\title{
The Growth of TiO2 Nanostructures Prepared by Anodization in Combination with Hydrothermal Method on the Ti Foil
}

\author{
Vatcharinkorn Mekla ${ }^{1, a}$, Charuwan Juisuwannathat ${ }^{2, b}$, and Udom Tipparach ${ }^{3,{ }^{*} \mathrm{c}}$ \\ 1,2 Program of Physics, Faculty of Science, Ubon Ratchathani Rajabhat University, Ubon Ratchathani \\ 34000, Thailand \\ ${ }^{3}$ Deparment of Physics, Faculty of Science, Ubon Ratchathani University, Ubon Ratchathani \\ 34000, Thailand
}

\begin{abstract}
We have investigated $\mathrm{TiO} 2$ nanostructures prepared by anodization in conjunction with hydrothermal method using $\mathrm{Ti}$ metal plates. The $\mathrm{TiO} 2$ nanoporus were fabricated by electrochemical anodization in a $\mathrm{NH} 4 \mathrm{~F} / \mathrm{EG} 4 / \mathrm{H} 2 \mathrm{O}$ electrolyte system. Ultrasonic wave was used to clean the surface of TiO2 nanoporus in the medium of water after completing the anodization. After drying in air, the nanoporusarrays were calcined at $450{ }^{\circ} \mathrm{C}$ for $2 \mathrm{~h}$ in air. The $\mathrm{TiO} 2$ nanostructures were converted by hydrothermal in air. The $\mathrm{TiO} 2$ nanostructures were characterized by $\mathrm{X}$-ray diffraction (XRD) and scanning electron microscopy (SEM). XRD patterns show the $\mathrm{TiO} 2$ anatase structure. SEM images indicate that the $\mathrm{TiO} 2$ structures depend on preparation temperatures. The density of $\mathrm{TiO} 2$ nanostructures increases as the time increases. The growth of $\mathrm{TiO} 2$ nanostructures was observed to be times dependence. The nanostructures are nanowires and nanospikes when the peraring time was $18 \mathrm{~h}$, nanoflowers when the preparing time was $24 \mathrm{~h}$. This approach provides the capability of creating patterned $1 \mathrm{D} \mathrm{TiO} 2$ nanowires at $18 \mathrm{~h}$. The diameter of $\mathrm{TiO} 2$ nanowires varies from $20 \mathrm{~nm}$ to $25 \mathrm{~nm}$ and length of several $250 \mathrm{~nm}$.
\end{abstract}

\section{Introduction}

$\mathrm{TiO}_{2}$ nanostructures have attracted great attention due to their unique ability to form a variety of nanostructures such as nanowires, nanoribbons, nanobelts, nanospheres and nanofibers and their properties. A special attention is focused on the $\mathrm{TiO}_{2}$ in the forms of nanorods and nanorod arrays vertically arranged with respect to the substrate because of their unique properties. The ordered $\mathrm{TiO}_{2}$ nanostructures are expected to enhance the performance of various technologically important devices such as electroluminescent devices [1], photocatalysis [2] gas sensors [3], and third generation of solar cells [4-5]. In this way, we prepared $\mathrm{TiO}_{2}$ on the $\mathrm{Zn}$ plates with 1D material constructed surface by this novel simple method. Recently, a new approach for the preparation of $\mathrm{TiO}_{2}$ nano tube on a patterned $\mathrm{Ti}$ foil has been developed by means of two step anodization process [6]. In the first step, potentiostatic anodization is applied and subsequently the developed $\mathrm{TiO}_{2}$ nano tube are detached from the titanium foil leaving behind a pattern, where thenew tubes grow during the second anodization step [7]. Titania nanotubes prepared by the two step potentiostatic potentiostatic method, are organized in well ordered films and free of surface defects [8]. The potentiostatic potentiostatic preparation of $\mathrm{TiO}_{2}$ nano tube has been further exploited for the guided anodization and fabrication of asymmetrical nanotubes onto patterned $\mathrm{Ti}$ foils treated by focused ionbeam lithography [9].The hydrothermal method is widely applied in titania nanotubes production because of its many advantages, such as high reactivity, lowenergy requirement, relatively non-polluting set-up and simple control of the aqueous solution [10]. The reaction pathway is very sensitive to the experimental conditions, such as $\mathrm{pH}$, temperature and hydrothermal treatment time, but the technique achieves a high yield of titania nanotubes cheaply and in a relatively simpler manner under optimized conditions. There are three main reaction steps in hydrothermal method such as generation of the alkaline titanate nanotubes substitution of alkali ions with protons and heat dehydration reactions in air [11]. The hydrothermal method is amenable to the preparation of $\mathrm{TiO}_{2}$ nanotubes with different crystallite phases such as the anatase, brookite, monoclinic and rutile phases [12].

\section{Experimental}

In the first step, preparation titanium foils (Jinsheng std.ASTMB265 Shaanxi company china, $0.3 \mathrm{~mm}$ ) were used as substrate for the anodization. Prior to the anodization, pieces (radius $1.8 \mathrm{~cm}$ ) of the Ti foils were ultrasonicated in acetone, 2-propanol and methanol for 10 min, then washed with water and dried under nitrogen. Anodization was performed in an appropriate

\footnotetext{
achokekana14@gmail.com, ${ }^{\mathrm{b}}$ cha16-thong@hotmail.co.th, ${ }^{*} \mathrm{c}$ udomt@hotmail.com
} 
electrochemical cell, made of teflon, at ambient temperature [13-14]. The working area was $10.18 \mathrm{~cm}^{2}$ and the distance between the anode (Ti foil) and the cathode (Pt mesh) was set at $1.5 \mathrm{~cm}$. The titanium foil were anodized at $50 \mathrm{~V}$ for $1 \mathrm{~h}$ in a fluorinated solution of ethylene glycol $\left(0.25 \mathrm{wt} \% \mathrm{NH}_{4} \mathrm{~F}, \quad 10 \mathrm{wt} \%\right.$ DI $\left.\mathrm{H}_{2} \mathrm{O}\right)$, followed by a brief cleaning with deionized (DI) water. The titanium nanostructures were further cleaned by dipping the anodes in a beaker of DI water under ultrasonication for 1-3 s. After drying in air, the nanotubes were calcined at $450{ }^{\circ} \mathrm{C}$ for $2 \mathrm{~h}$ in air. In the second step, the synthesis method of nanostructure was basically the same as in previous works [15]. A commercial, $\mathrm{TiO}_{2}$ powder (commercial; a mixture of crystalline rutile and anatase phases) was used as a starting material. In a typical synthesis, $1 \mathrm{~g}$ of $\mathrm{TiO}_{2}$ powder wascrushedwith $25 \mathrm{~mL}$ of $10 \mathrm{M} \mathrm{NaOH}$ aqueous solution were putt into a teflon- lined stainless autoclave and then heated at $180^{\circ} \mathrm{C}$ for $6 \mathrm{~h}, 12 \mathrm{~h}, 18 \mathrm{~h}, 24 \mathrm{~h}$ and 46 h. The samples were cooled down to room temperature.. The treated samples were washed thoroughly with DI water and $0.1 \mathrm{~mol} / \mathrm{L} \mathrm{HCl}$ aqueous solutions until the $\mathrm{pH}$ value of the washing solution lower than 7 and dried at 60 ${ }^{\circ} \mathrm{C}$. The structural and chemical natures of the obtained materials were studied using X-ray diffraction (XRD), scanning electron microscopy (SEM).

\section{Results and discussion}

The samples were synthesized under different periods of time and were assinged as 6h, 12h, $18 \mathrm{~h}, 24 \mathrm{~h}$, and $46 \mathrm{~h}$. Ti-anealed (substrates for the anodization) and $\mathrm{TiO}_{2}$ crystal structure were used for comparision purpose. XRD patterns are shown in Fig. 1.

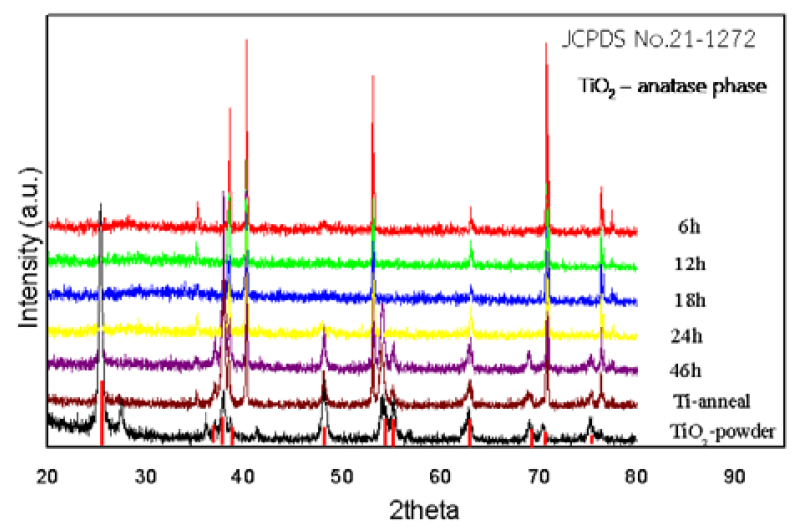

Figure 1. XRD patterns of the samples were synthesized under at time and were as singed as $6 \mathrm{~h} 12 \mathrm{~h}, 18 \mathrm{~h}, 24 \mathrm{~h}, 46 \mathrm{~h}$, Ti-anneal (substrate for the anodization) and $\mathrm{TiO}_{2}$ powder.

All the peaks are sharp and strong in intensity indicating the highly crystalline in nature of the reaction products. $\mathrm{TiO}_{2}$ anatase phase was observed in the XRD patterns and diffraction data in agreement with JCPDS card of $\mathrm{TiO}_{2}$ (JCPDS No. 21-1272), which indicated that the samples on the substrates were $\mathrm{TiO} 2$ and partially the crystalline of Ti-annealed substrates. The peaks located at 25.4, 37.8, $48.0,54.5^{\circ}$ correspond to the (101), (004), (200), (105 and 211) planes of the anatase phase (JCPDS 21-1272), and the peaks located at $27.5,36.1,54.4^{\circ}$ correspond to the (110), (101), (211) planes of the rutile phase (JCPDS 211276), respectively.
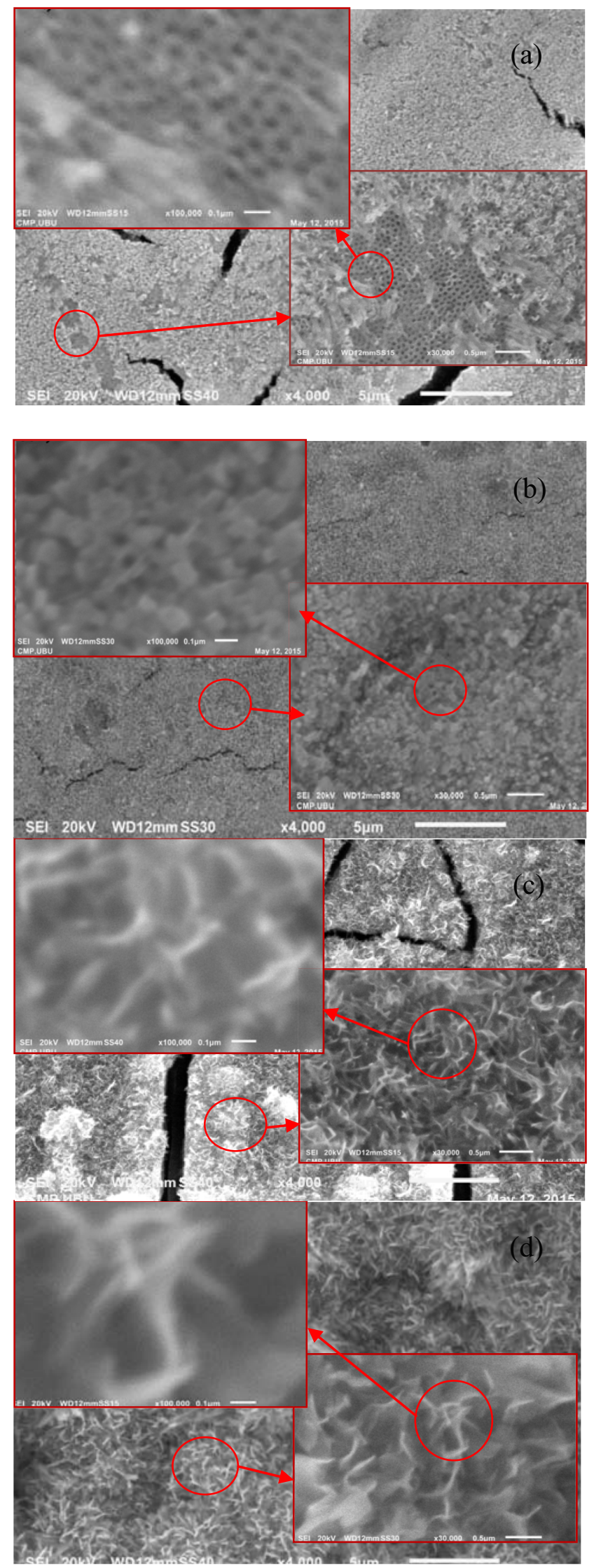


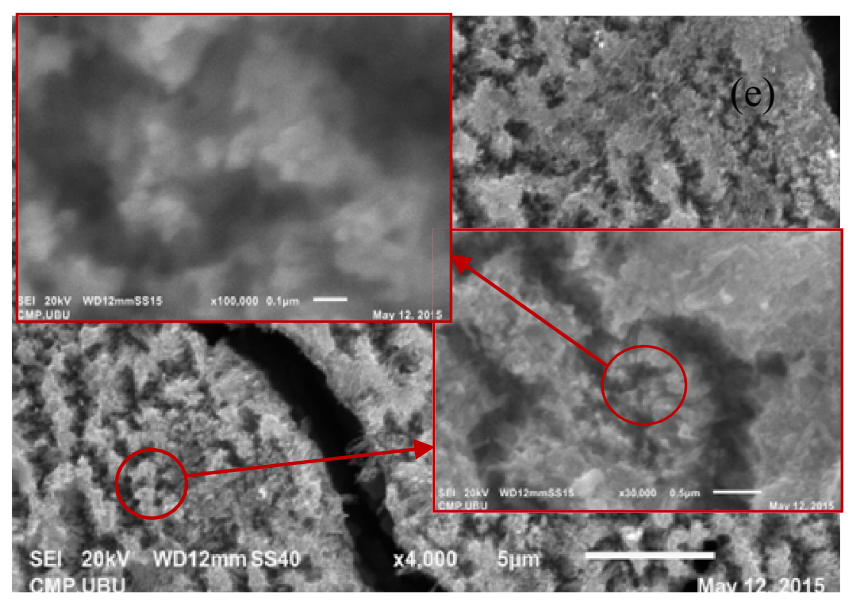

Figure 2. The side view SEM images of the samples,prepared using the heated at $180{ }^{\circ} \mathrm{C}$ for (a) $6 \mathrm{~h} \mathrm{(b)}$ $12 \mathrm{~h} \mathrm{(c)} 18 \mathrm{~h} \mathrm{(d)} 24 \mathrm{~h}$ and (e) $46 \mathrm{~h}$.

The top-view SEM images clearly show that anodized titania nanotubes with hydrothermal method are nanostructures. Their crystals form porosity and nanotube samples prepared by anodization. The pictures also show that when longer period of time of hydrothermal process was used toanneal, the nanocrystal structures increase. We found that the crystal structures become nanowires and nanospike. The nanowires and nanospikesare denser and vertically more scattered whenannealing temperatures of the hydrothermal process of $180^{\circ} \mathrm{C}$ for 18 hand $24 \mathrm{~h}$.

\section{Conclusion}

$\mathrm{TiO}_{2}$ nanowires and nanospikeswere successfully fabricated by anodization in combination with hydrothermal method of Ti foil at $180^{\circ} \mathrm{C}$ for $18 \mathrm{~h}$ and 24 $\mathrm{h}$. The structures depend on time and the temperature of hydrothermal process. The diameter of $\mathrm{TiO}_{2}$ nanowires varies from $20 \mathrm{~nm}$ to $50 \mathrm{~nm}$ and length of several 100 micrometers.

\section{Acknowledgements}

This work is supported by Program of Physics, Faculty of Science, UbonRatchathaniRajabhatUniversity and UbonRatchathani University. The authors gratefully thank them.

\section{References}

1. P.Wongwanwattana, P. Krongkitsiri, P. Limsuwan, and U. Tipparach: Ceramics InternationalVol.38(2012), p.517-519

2. S.H. Kang, S.H. Choi, M.S. Kang, J.Y. Kim, H.S. Kim, T. Hyeon and Y.E. Sung: Adv. Mater Vol.20 (2008), p.54

3. Y.Y. Zhang, W.Y. Fu and H.B. Yang:Surf. SciVol. 254 (2008),p.5545-5547

4. U.Tipparach,andP.Limsuwan: Adv. Materials Research Vol. 93(2010), p. 263-267

5. Y.S. Chen, J.C. Crittenden and S. Hackney: Sci. TechnolVol.39 (2005), p.1201-1208

6. S. Li, G. Zhang, D. Guo, L. Yu, and W. Zhang: Journal of Physical Chemistry C Vol. 113(2009), p. 12759-12765

7. Y. Shin and S. Lee:NanoLett. Vol.8 (2008), p.3171

8. B. Chen, K. Lu, and J. A. Geldmeier:Chemical Communications Vol. 47(2011), p. 10085-10087

9. B. Chen, K. Lu and Z. Tian: Langmuir, Vol.27(2011), p. 800-808

10. C.K. Lee, C.C. Wang, M.D. Lyu, L.C. Juang, S.S. Liu andS.H Hung: J. Colloid Interface Sci.Vol.316(2007), p.562-569

11. H.S.Hafez: Mater. Lett. Vol.63(2009), p.1471-1474

12. B.X.Wang, D.F Xue, Y.Shi and F. H. Xue:Titania 1D nanostructured materials:synthesis, properties and applications. In: Prescott, W.V., Schwartz, A.I. (Eds.),Nanorods, Nanotubes and Nanomaterials Research Progress. New Nova SciencePublishers Inc., New York, (2008), p. 163-201

13. R.Vongwatthaporn and U. Tipparach:Applied Mechanics and Materials, Vol.749 (2015), p.191196

14. W.Sangworn, P.Krongkitsiri, T. Saipin, B. Samran, and U. Tipparach: Advanced Materials Research, Vol.741(2013), p.84-89

15. J. Jitputti, S. Pavasupree, Y. Suzuki, S. Yoshikawa: Appl. Phys. Vol. 47 (2008), p.751-756 